\title{
Special issue on emerging challenges in multi-agent sensing, control and optimization
}

\author{
Bijoy Kumar Ghosh ${ }^{1,2} \cdot$ Jiangping $\mathrm{Hu}^{2} \cdot{\text { Xiaoming } \mathrm{Hu}^{3}}^{3}$
}

Received: 15 October 2020 / Accepted: 19 October 2020 / Published online: 26 November 2020

(c) South China University of Technology, Academy of Mathematics and Systems Science, CAS and Springer-Verlag GmbH Germany, part of Springer Nature 2020

Up to now, a large number of theoretical achievements and application scenarios have been obtained in sensing, control and optimization of multi-agent systems. However, challenges are still emerging from the rapid development of artificial intelligence and urgent need for distributed computing. The aim of the special issue is to present in a systematic way some new results in the fields of distributed sensing, control and optimization of cyber-physical systems, particularly in resource allocation, machine learning, observer and filtering, consensus and constrained control.

The collection consists of nine research papers and one letter, and can be divided into five groups: the first group is about distributed optimization. Y. Wang et al. studied a resource allocation problem using stochastic mirror descent algorithm. The second group focuses on machine learning. J. Okae et al. presented a robust statistical approach to noise detection and refinement of Winner-Takes-All (WTA) disparity maps that improves the stereo labeling performances of convolutional neural networks (CNNs). A distributed policy evaluation was studied in the paper by $\mathrm{X}$. Zhao et al. for multi-agent reinforcement learning using an inexact ADMM (alternating directions method of multipliers) algorithm. Z. Peng et al. developed a distributed multi-agent temporal-difference (TD) learning framework for value function approximation using all the neighbors' information. The third group includes two papers about distributed observers and filters. D. Xu et al. proposed a class of distributed tracking observers with locally jointly observable sensors. A consistent Kalman-type nonlinear filter was applied to the track-to-track association analysis of multi-targets with uncertain dynamics in the paper by X. He et al. The fourth group includes two papers on consensus. A privacy-preserving consensus problem was solved in the paper by S. Zhang and M. Dahleh using a Shamir's secret sharing scheme. J. Wei et al. proposed a finite-time consensus control strategy based on a direction preserving signum method. The last group of this collection includes two papers with cyber-physical background. B. K. Ghosh and B. Athukorallage constructed a single Riemannian metric to study a combined optimal gaze rotation for two eyes and an optimal external torque was obtained for eye movement maneuvers. $\mathrm{X}$. Yin introduced recent advances on formal methods for safety and security of cyber-physical systems.

Finally, the guest editors would like to thank Tiefeng Zou, Editorial Assistant, and Yiguang Hong, Editor-in-Chief of the Journal of Control Theory and Technology, for their valuable suggestions and help in the process.

\section{Guest editors:}

Bijoy Kumar Ghosh

Jiangping $\mathrm{Hu}$

Xiaoming $\mathrm{Hu}$
Jiangping $\mathrm{Hu}$

hjp_lzu@163.com

Texas Tech University, Lubbock, USA

2 University of Electronic Science and Technology of China, Chengdu, China

3 KTH Royal Institute of Technology, Stockholm, Sweden 


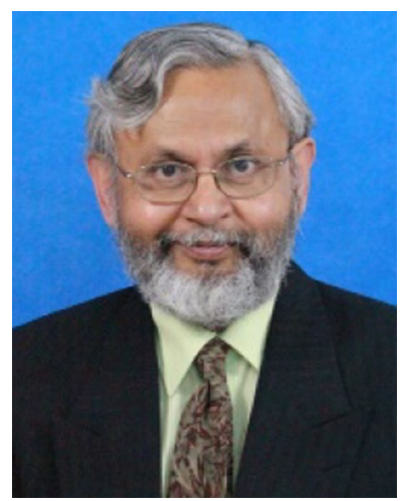

Bijoy Kumar Ghosh received the Ph.D. degree in Harvard University 1983 . From 1983 to 2007 , he was with the Department of Electrical and Systems Engineering, Washington University. Currently he is the Dick and Martha Brooks Regents Professor of Mathematics and Statistics at Texas Tech University, Lubbock, TX, USA. He became an IEEE Fellow in 2000, and a Fellow of the International Federation on Automatic Control in 2014. His research interests include biomechanics, cyberphysical systems and control problems in rehabilitation engineering.

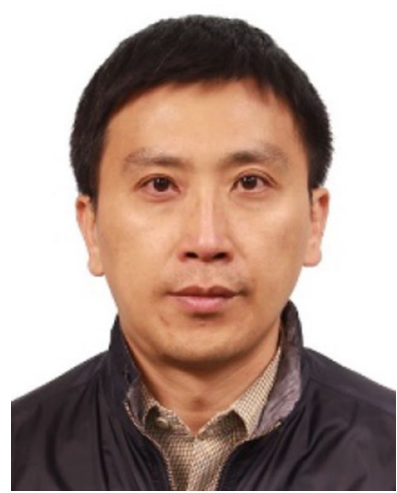

Jiangping $\mathrm{Hu}$ received the B.S. degree in applied mathematics and the M.S. degree in computational mathematics from Lanzhou University, Lanzhou, China, in 2000 and 2004, respectively, and the Ph.D. degree in modelling and control of complex systems from the Academy of Mathematics and Systems Science, Chinese Academy of Sciences, Beijing, China, in 2007. He has held various positions with the Royal Institute of Technology, Stockholm, Sweden, The City University of Hong Kong, Hong Kong, Sophia University, Tokyo, Japan, and Western Sydney University, Sydney, NSW, Australia. He is currently a
Professor with the School of Automation Engineering, University of Electronic Science and Technology of China, Chengdu, China. His current research interests include multi-agent systems, social dynamics, and sensor networks. Dr. Hu has served as an Associate Editor of journal Kybernetika since 2016.

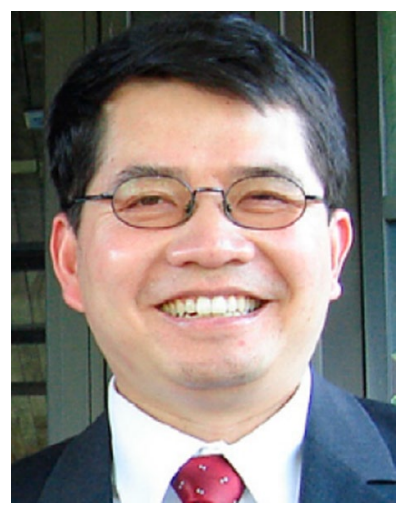

Xiaoming Hu received the B.S. degree from the University of Science and Technology of China in 1983, and the M.S. and $\mathrm{Ph} . \mathrm{D}$. degrees from the Arizona State University in 1986 and 1989 respectively. He served as a research assistant at the Institute of Automation, the Chinese Academy of Sciences, from 1983 to 1984 . From 1989 to 1990 he was a Gustafsson Postdoctoral Fellow at KTH Royal Institute of Technology, Stockholm, where he is currently a professor of Optimization and Systems Theory. His main research interests are in nonlinear control systems, nonlinear observer design, sensing and active perception, motion planning, control of multi-agent systems, and mobile manipulation. 